\title{
Colorectal Disease
}

\section{An international pragmatic randomised controlled trial to compare a single use negative pressure dressing versus a surgeon's preference of dressing to reduce the incidence of surgical site infection following emergency laparotomy: the SUNRRISE Trial Protocol}

\begin{tabular}{|c|c|}
\hline Journal: & Colorectal Disease \\
\hline Manuscript ID & CDI-01290-2020.R1 \\
\hline Wiley - Manuscript type: & Trial Protocol \\
\hline $\begin{array}{r}\text { Date Submitted by the } \\
\text { Author: }\end{array}$ & $n / a$ \\
\hline Complete List of Authors: & $\begin{array}{l}\text { Wilkin, Richard; University of Birmingham, Institute of Cancer and } \\
\text { Genomic Studies }\end{array}$ \\
\hline Specialty area: & surgery, other \\
\hline Abstract: & $\begin{array}{l}\text { Background: } \\
\text { Surgical site infection (SSI) is a common complication following } \\
\text { emergency laparotomy occurring in around } 25 \% \text { of patients in UK } \\
\text { practice. The use of single use negative pressure dressings (SUNPDs) for } \\
\text { these wounds has been proposed as a prophylactic method of reducing } \\
\text { the rate of SSI. } \\
\text { Method: } \\
\text { The Single Use Negative pRessure dressing for Reduction In Surgical site } \\
\text { infection following Emergency laparotomy (SUNRRISE) study is an } \\
\text { international, multicentre, pragmatic, phase III randomised controlled } \\
\text { trial (RCT) with internal feasibility phase. The primary aim is to } \\
\text { determine if a single use negative pressure dressing (SUNPD) reduces } \\
\text { surgical site infection (SSI) at } 30 \text { days post-operatively. Patients will be } \\
\text { randomised in a } 1: 1 \text { ratio to either a SUNPD or to receive a dressing of } \\
\text { the surgeon's preference. Outcome assessors will be blinded to } \\
\text { treatment allocation. The primary outcome measure is SSI within } 30 \\
\text { days of surgery as defined by the Centers for Disease Control criteria. A } \\
\text { total of } 840 \text { patients will be required to detect a relative reduction of } \\
40 \% \text { in SSI rates (from } 25 \% \text { to } 15 \% \text { ) with } 90 \% \text { power accounting for } \\
20 \% \text { attrition rate. } \\
\text { Discussion: } \\
\text { SUNRRISE is an international, multicentre RCT evaluating the } \\
\text { prophylactic use of SUNPD in primary closed emergency laparotomy } \\
\text { wounds for the reduction of SSI. Our hypothesis is that a SUNPD is } \\
\text { superior to the surgeon's preference of dressing in reducing surgical site } \\
\text { infections at } 30 \text { days. These findings may influence dressing choice } \\
\text { following emergency abdominal surgery in the future. }\end{array}$ \\
\hline
\end{tabular}


SCHOLARONE $^{\text {TM }}$
Manuscripts

Manuscripts 
An international pragmatic randomised controlled trial to compare a single use negative pressure dressing versus a surgeon's preference of dressing to reduce the incidence of surgical site infection following emergency laparotomy: the SUNRRISE Trial Protocol

The SUNRRISE Study Group on behalf of the Northwest Research Collaborative and the West Midlands Research Collaborative*

${ }^{*}$ Collaborating authors are listed in Appendix 1

Corresponding author: Richard Wilkin, Academic Clinical Lecturer, University of Birmingham, r.wilkin@bham.ac.uk

ISRCTN reference number: 17599457

ACTRN reference number: 12619000496112

Protocol version 3.0 (05-Aug-2020)

Financial support:

SUNRRISE is funded by the Research for Patient Benefit program from the National Institute for Health Research (NIHR) in the UK and the Medical Research Future Fund (MRFF) International Clinical Trials Collaboration Program in Australia. The interventional arm dressings are supplied for the trial by Smith \& Nephew who also distribute the dressings to the sites involved. At each site the company is providing dressing application and management training to the local teams in line with their usual training support for sites using their dressings. This training is for general use of their PICO dressings and not specific to the trial and does not cover any part of the trial protocol or participant management. SUNRRISE is an investigator initiated study, the design, management, analysis and reporting are entirely independent from the funders, including Smith \& Nephew. The academic investigators retain full independence and autonomy for study conduct and reporting. This paper presents independent research funded by the NIHR under its Research for Patient Benefit (RfPB) Programme (PB-PG-0416-20045) and the MRFF International Clinical Trials Collaboration (ICTC) Program (MRFF1179938). The views expressed are those of the author(s) and not necessarily those of the NIHR, the Department of Health and Social Care or the Commonwealth of Australia.

Primary Trial Sponsor: University of Birmingham (UK)

Sponsor's Reference: RG_17-239

Address: Research Governance Team, University of Birmingham, Birmingham, B15 2TT

Email: researchgovernance@contacts.bham.ac.uk

Secondary Trial Sponsor: University of Newcastle (Australia).

Address: Research and Innovation Services, University of Newcastle, Callaghan, 2308

Email: research-grants@newcastle.edu.au 
Organisational structure and responsibilities

Trial Management Group (UK): James Brown, Hamish Clouston, Peter Coe, Rui Duarte, Sarah Duff, Rebecca Fish, Paula Ghaneh, James Glasbey, Natalie Ives, Elisabeth Johnstone, Manjinder Kaur, Laura Magill, Samir Mehta, Thomas Pinkney, Kellie Platt, Peter Pockney, Hema Sekhar, Yash Sinha, Martyn Stott, Richard Wilkin.

Trial Management Group (Australia): Hossein Afzali, Thomas Arthur, Amanda Dawson, Nagendra N Dudi-Venkata, Natasha Egoroff, Madelyn Gramlick, Yick Ho Lam, Vijayaragavan Muralidharan, Sean SW Park, Peter Pockney, Toby Richards, Tarik Sammour, Bree Stephensen, Sean Stevens, Uyen Giao Vo, David Watson, Richard Wilkin.

Data Monitoring Committee: Nigel Hall (Chair), Joanne Dumville, Ly-Mee Yu.

Trial Steering Committee: Matt Costa (Chair), Neil Corrigan, Marcos Gomez Ruiz, Lesley Osborn (Patient Representative).

Acknowledgement: We would like to acknowledge the input from Professor Jon Gani (University of Newcastle (Australia)), Professor Michael Solomon (University of Sydney), Professor Sandy Heriott (University of Melbourne), Professor John Attia (University of Newcastle), Dr Alison Blatt (John Hunter Hospital) and Dr Ani Deshpande (Westmead Childrens' Hospital) who have provided local trial oversight in Australia.

Conflicts of interest: The authors of this protocol paper do not have any conflicts of interest to declare.

Key words: Surgical site infection, wound infection, topical negative pressure dressing, randomised controlled trial, PICO dressing.

Word count: 3825 


\section{Abstract}

Background: Surgical site infection (SSI) is a common complication following emergency laparotomy occurring in around $25 \%$ of patients in UK practice. The use of single use negative pressure dressings (SUNPDs) for these wounds has been proposed as a prophylactic method of reducing the rate of SSI.

Method: The Single Use Negative pRessure dressing for Reduction In Surgical site infection following Emergency laparotomy (SUNRRISE) study is an international, multicentre, pragmatic, phase III randomised controlled trial (RCT) with internal feasibility phase. The primary aim is to determine if a single use negative pressure dressing (SUNPD) reduces surgical site infection (SSI) at 30 days post-operatively. Patients will be randomised in a 1:1 ratio to either a SUNPD or to receive a dressing of the surgeon's preference. Outcome assessors will be blinded to treatment allocation. The primary outcome measure is SSI within 30 days of surgery as defined by the Centers for Disease Control criteria. A total of 840 patients will be required to detect a relative reduction of $40 \%$ in SSI rates (from $25 \%$ to $15 \%$ ) with $90 \%$ power accounting for $20 \%$ attrition rate.

Discussion: SUNRRISE is an international, multicentre RCT evaluating the prophylactic use of SUNPD in primary closed emergency laparotomy wounds for the reduction of SSI. Our hypothesis is that a SUNPD is superior to the surgeon's preference of dressing in reducing surgical site infections at 30 days. These findings may influence dressing choice following emergency abdominal surgery in the future. 


\section{Background}

An emergency laparotomy is a major operation with the risk of significant morbidity. One of the most common complications of this operation is a Surgical Site Infection (SSI). Reported rates of SSI vary across the literature but several large studies have reported SSI rates in excess of 25\% $\%^{1,2}$. In England, there are over 30,000 emergency laparotomies performed each year. Emergency abdominal surgery is undertaken by a variety of abdominal, breast and vascular surgeons in the UK and Australia, and over half of emergency laparotomies are performed by colorectal surgeons ${ }^{3}$. Morbidity from these operations represent a significant burden upon healthcare providers and patients $^{4,5}$. SSI places significant physical and psychological burden on patients. A severe SSI will require long-term nursing care, often in the community, and can prevent the patient from returning to work and re-integrating into their normal routine and social circles. It is also well established that SSI increases the cost of inpatient care significantly; the average cost is estimated at $£ 3500$ per patient ${ }^{6}$.

Single use negative pressure wound dressings (SUNPD) have been proposed as a method for reducing the incidence of SSI. Several randomised controlled trials (RCTs) have suggested that these dressings are effective for SSI reduction in closed wounds following limb surgery ${ }^{7,8}$. However, the most recent multicentre RCT involving patients following surgery for traumatic lower limb fractures does not show any benefit from the dressings $^{9}$. There are an increasing number of studies investigating SUNPDs in abdominal surgery. The majority of these are non-randomised and there are no studies exclusively investigating emergency laparotomies. A guideline from the World Health Organisation (WHO) Infection Prevention committee suggests use of the dressings in high-risk wounds ${ }^{10}$. However, its scope is limited by the inherent bias of basing this decision on mainly non-randomised studies ${ }^{11-13}$. A Cochrane meta-analysis published in 2020 included 44 RCTs of different types of surgery and found "moderate-certainty evidence" that SUNPDs reduce the incidence of SSI ${ }^{14}$. However, a meta-analysis of only laparotomies (elective and emergency) found no significant difference between negative pressure wound therapy and standard dressings in the rate of $\mathrm{SSI}^{15}$. The high incidence of SSI following emergency laparotomy means any intervention which could reduce SSI could deliver a significant benefit to patients and health care providers. To date there have been no randomised trials investigating the effectiveness of SUNPD in this context.

The SUNRRISE trial aims to evaluate whether SUNPD are effective in reducing SSI within 30 days of surgery in patients who have undergone an emergency laparotomy with primary wound closure. 


\section{Methods}

\section{Ethics and reporting}

This study protocol is reported in accordance with the guidance set out in the SPIRIT statement ${ }^{16}$. SUNRRISE has obtained ethical approval in England \& Wales, Scotland and Australia: 18/YH/0322 (England and Wales), 19/SS/0065 (Scotland), 2019/ETH00189 (Australia).

\section{Trial design}

The SUNRRISE Trial is an international, multicentre, pragmatic, phase III RCT with an internal feasibility stage comparing the use of SUNPD with the surgeon's preferred dressing. The primary objective of the study is to determine if the use of a SUNPD in adult patients undergoing emergency laparotomy reduces SSI within 30 days compared to surgeon's preference of dressing (which may be conventional occlusive dressings, skin glue or no dressing, but not another SUNPD).

SUNRRISE will be open to any hospital that performs emergency laparotomies in either the United Kingdom or Australia. No centre volume restrictions will be imposed. The trial aims to recruit participants from over 25 sites.

SUNRRISE is a pragmatic trial and does not mandate or prohibit any specific measures that surgeons use in routine practice to prevent SSI, for example prophylactic antibiotic administration, specific antimicrobial skin preparations or intraoperative wound washing. Use of these measures will be collected in the trial Case Report Forms (CRFs). In line with the pragmatic design of the study, there are no restrictions on the type of emergency laparotomy that a patient may undergo, how the incision is closed (apart from that the incision should not be closed with glue) or the post-operative care of the patient (except for the type of dressing used in the immediate post-operative period).

\section{Eligibility Criteria}

The inclusion criteria for the trial are;

- Patients undergoing emergency (non-elective) laparotomy

- Procedures with an incision of at least $5 \mathrm{~cm}$

- Operations where the skin is closed primarily

- Patients aged at least 16 years in UK, or at least 18 years in Australia

- Patients able to provide informed consent or, in the UK only, consultee/representative provides assent/consent if a patient temporarily lacks capacity

- Patients willing and able to undergo follow-up at 30 days post-op

The exclusion criteria for the trial are;

- Abdominal surgery within the preceding three months from the date of randomisation 
- Expected return to theatre for reopening of the laparotomy wound within 30 days

\section{Internal feasibility}

The aims of the feasibility study were to assess the ability of sites to recruit patients to the trial, adherence to the trial allocation and the loss-to-follow-up rate. The number of participants to be recruited in the six month feasibility period was 70 patients from at least 5 sites. The decision to continue the trial was guided by pre-defined stop-go criteria using a traffic light system (see Table 1). In addition to these stop-go criteria, there was an assessment of safety by the Data Monitoring Committee (DMC). At the end of the feasibility phase, the 'green' criteria were all met and there were no safety issues, so the Trial Steering Committee (TSC) were happy to support the continuation of the trial.

\section{Outcomes}

The primary outcome measure is SSI within 30 days of surgery, defined as a composite of superficial and deep incisional SSI from the internationally accredited Centers for Disease Control (CDC) criteria. The CDC criteria are widely used and validated in trial settings, and are consistent with the Public Health England criteria for SSI used in national surgical site infection audits in the United Kingdom. The following definition will be used to identify an SSI:

- The infection must occur within 30-days of the index operation

AND

- The patient must have at least one of the following:

- Purulent drainage from the wound

- Organisms are detected from a wound swab

- Wound opened spontaneously or by a clinician AND, at the surgical wound, the patient has at least one of: pain or tenderness; localised swelling; redness; heat; systemic fever $\left(>38^{\circ} \mathrm{C}\right)$

- Diagnosis of SSI by a clinician or on imaging

The 30 day wound assessment will be conducted in-person, or if necessary by video call (providing a visualisation of the wound) by a trained wound assessor blinded to treatment allocation. The assessors will hold medical or nursing qualifications; for example doctors, nurses, advanced care practitioners or physician associates. All research team members who are undertaking wound assessments will be required to undertake the online module for the diagnosis of SSI. This will ensure standardisation of diagnosis and can be accessed via the trial website (www.birmingham.ac.uk/SUNRRISE).

Secondary outcome measures include:

- Length of hospital stay after surgery 
- Wound complications within 30 days post-surgery as graded by Clavien-Dindo scale $^{17}$

- Hospital re-admission for wound related complications within 30 days

- Health-related quality of life using the Short Form-12 (SF-12) and EuroQuol-5 Dimension-5 Level (EQ-5D-5L)

- Pain at the site of the primary laparotomy using a 10-point visual analogue scale

- Serious adverse events up to 30 days

- Cost-effectiveness

- Patient and health professional acceptability of the dressing

\section{Consent}

The majority of patients undergoing an emergency laparotomy will be able to provide fully informed consent. There are, however, a proportion of patients who meet the inclusion criteria for the study who are either unable to provide full consent or are not able to consent at all due to a temporary impairment resulting from the indication for their emergency laparotomy. Patients may be unconscious, critically unwell, distracted by pain or anxiety, or have received large doses of opiates for pain relief, potentially affecting their ability to process information. The methods of gaining consent for inclusion in the study are different for patients who are able to provide consent and those who are not. The law around recruitment of patients that lack capacity is governed under the Mental Capacity Act in England and Wales, by Adults with Incapacity (Scotland) Act in Scotland and by State/Territory law in Australia. A flowchart for the consent process is found in Figure 1.

If patients are able to provide informed consent, they will be provided with a 'Patient Information Sheet' and asked to complete a trial specific 'Informed Consent form' and the standard procedures around consent will be performed.

If patients do not have capacity to provide informed consent due to a temporary impairment, they will potentially be eligible to participate in the trial. If they are in England, the patient must have a Personal Consultee present. This person is asked to provide their assent to the patient participating in the trial. In Scotland, the patient must have a Legal Representative (nearest relative) present. This person is asked to provide consent on the patient's behalf. In both cases, as soon as the patient is considered able to provide informed consent, the trial will be explained to them, an information sheet provided and their written informed consent will be sought.

Sites in Australia are not using the above delegated consent processes. The law that governs this area varies across States and Territories making it difficult to achieve consistency across the country. Therefore, a pragmatic decision was made that if a patient in Australia is unable to provide informed consent, they will not be eligible to be included in the trial.

\section{Randomisation}

Patients will be consented before their operation. If the patient agrees to enter the trial, they will be asked to complete a trial consent form and a baseline quality of life booklet which contains both the EQ-5D-5L and the SF-12 questionnaires. 
At the end of the patient's operation, eligibility will be confirmed. If the patient is still eligible when skin closure is commenced, they will be randomised into SUNRRISE. Patients will be randomised at the level of the individual in a 1 to 1 ratio to either SUNPD or surgeon's preference of dressing. A minimisation algorithm will be used to ensure balance in treatment allocation over the following variables: recruitment centre, presence of a stoma (Yes, No) and the degree of contamination (Clean, Cleancontaminated, Contaminated, Dirty) identified during the operation. So that the randomisation process is not completely deterministic, a 'random element' will be included in the algorithm. Randomisation will be provided by a single, central secure online or automated telephone randomisation system. Both the online and telephone systems will be available 24 hours a day, 7 days a week.

\section{Intervention}

The intervention is the application of a SUNPD to the incision immediately after it has been closed whilst still in theatre. The dressing used in SUNRRISE is the PICO7 Single Use Negative Pressure Wound Therapy System ${ }^{18}$. This dressing should remain in place for up to 7 days post-operatively, or until the patient is discharged from hospital (whichever is sooner). Some patients will have a single dressing that stays in place for seven days, others will require this dressing to be changed for a variety of reasons. If the dressing requires changing, it can be changed as many times as needed depending on whether the research team have surplus dressing pads.

\section{Control arm}

The control arm is the surgeon's preference of dressing. This is defined as any type of occlusive dressing (including glue) or no dressing at all. If a dressing is used it must not be a silver, honey or iodine based dressing. This arm also excludes any type of topical negative pressure dressing.

\section{Assessment schedule}

At day 7 or at discharge from hospital (whichever is sooner), the patient and wound will be assessed and the day 7 wound assessment CRF will be completed. The participant will be asked to rate their wound pain using a 10-point visual analogue scale. The participant will also be asked to complete the EQ-5D-5L and SF-12 Quality of Life questionnaires. If the participant is discharged before day 7 (and hence the wound assessment is being performed before day 7), the participant will be asked to complete the EQ-5D-5L and SF-12 at day 7 post-operatively using the forms in the 'Patient Diary'.

Between the point of discharge and when the patient has their 30 day assessment, the patient will be asked to complete a 'Patient Diary'. This involves a daily selfreported assessment of data related to the patient and their wound. On days 7, 14, 21 and 30, the patient completes an EQ-5D-5L questionnaire and on days 7 and 30 the participant completes an SF-12 questionnaire. Once discharged, patients will be contacted by the research team at their centre on a weekly basis to remind them to complete their diary. At the end of the diary, the patient is asked to complete the 
Wound Healing Questionnaire, which is then repeated by the clinician performing the 30 day wound assessment. The data will be used to provide external validation of this tool for wound assessment.

At day 30, a review will take place with a blinded wound assessor, and the wound assessed for an SSI according to the CDC criteria. As part of this wound assessment, patients will be asked to rate their wound pain using a 10-point visual analogue scale. The patient will also be asked to complete the Quality of Life questionnaires, EQ-5D$5 \mathrm{~L}$ and SF-12, if they have not already done so.

A schematic diagram of all of the potential assessments that a patient may undergo is shown in Figure 2. A flow chart of the activities for sites to complete during the care pathway of a patient can be found in Appendix 2. The full protocol and the CRFs for the trial can be found online on the trial website at www.birmingham.ac.uk/SUNRRISE.

\section{Blinding}

It is not possible to blind the patient to their trial allocation, but the 30 day wound assessor will be blinded to the trial allocation. The importance of blinding will be explained to patients and they will be asked to not inform the wound assessor of their treatment arm.

\section{Sample size}

The justification for the sample size is based on data from the ROSSINI trial ${ }^{1}$, which reported an SSI rate of $25 \%$ in the control group. To detect a relative reduction of $40 \%$ in SSI rates (i.e. from $25 \%$ to $15 \%$, so $10 \%$ absolute difference) between groups using the standard method of difference between proportions (2-sided) with $90 \%$ power and a type I error rate of $5 \%$ (i.e. $\alpha=0.05$ ), requires 336 participants per group to be randomised, so 672 in total. Assuming and adjusting for a $20 \%$ attrition/loss to followup rate (based on the death rate in this population being approximately $10 \%$ at 30 days; further drop out 10\%), 840 participants (420 per group) will need to be recruited.

The $40 \%$ reduction correlates with the relative reduction that was expected in other large HTA funded SUNPD trials, such as $\mathrm{WHIST}^{9}$ and it is based on the available literature regarding the potential effect of these dressings.

\section{Statistical Analysis}

A separate Statistical Analysis Plan (SAP) will be produced and will provide a more comprehensive description of the planned statistical analyses. A brief outline of the analyses is given below.

The primary comparison groups will be composed of those who are randomised to SUNPD versus those randomised to the surgeon's preferred dressing. All analyses will be based on the intention to treat principle. For all major outcomes, summary statistics and differences between groups (e.g. mean differences, relative risks) will be presented, with $95 \%$ confidence intervals and $p$-values from two-sided tests also 
given. Analyses will be adjusted for the minimisation variables (recruitment centre, presence of a stoma and the degree of contamination) and baseline scores (where available). Recruitment centre will be included in the model as a random effect; other variables will be included as fixed effects. A p-value of $<0.05$ will be considered statistically significant, and there will be no adjustment for multiple testing.

\section{Primary outcome analysis}

The primary outcome is SSI within 30 days of surgery, as defined by the internationally accredited CDC criteria. This is a binary outcome (i.e. yes/no). The number and percentage of patients reporting an SSI within 30 days of surgery will be reported by treatment group. An adjusted relative risk and $95 \%$ confidence interval will be estimated from a mixed effects log-binomial regression model. A risk difference and $95 \%$ confidence interval will also be provided. Statistical significance of the treatment group parameter will be determined from the $\mathrm{p}$-value generated by the model.

\section{Secondary outcomes analysis}

The secondary outcomes for this trial include a combination of continuous and categorical data items.

\section{Categorical outcomes (Wound complications, Hospital readmission for wound related complications, Serious Adverse Events)}

For binary secondary outcomes, the number and percentage of patients reporting each outcome will be reported by treatment group. An adjusted relative risk and $95 \%$ confidence interval will be estimated from a mixed effects log-binomial regression model.

\section{Continuous outcomes (Length of hospital stay after surgery, SF-12, EQ-5D-5L, visual analogue scale for pain)}

Length of hospital stay will be summarised using means and standard deviations, and compared between groups using mixed effects linear regression models to obtain an adjusted mean difference and $95 \%$ confidence interval. If data are skewed, then data will be summarised using medians and interquartile ranges, and an unadjusted difference between medians will be reported. The SF-12, EQ-5D-5L and assessment of pain at the site of the primary laparotomy will also be summarised using means and standard deviations. The data at both day 7 and day 30 will be compared between groups using mixed effects linear regression models to obtain an adjusted mean difference and $95 \%$ confidence interval. Data for the EQ-5D-5L is collected at baseline and days $7,14,21$ and 30 , and so as a secondary analysis, this outcome will also be analysed using a mixed effects repeated measures model.

\section{Planned subgroup analyses}

Subgroup analyses will use the same variables as those in the minimisation algorithm (with the exception of centre), as well as the operative procedure and country. Subgroup analyses will be limited to the primary outcome. The effects of these subgroups will be examined by including a treatment group by subgroup interaction parameter in the regression model. The results of subgroup analyses will be treated with caution and will be used for the purposes of hypothesis generation only. 
The health economic analysis will determine the costs and benefits of SUNPD versus the surgeon's preferred dressing. Separate analyses will be undertaken in Australia and the UK. In the UK, the economic evaluation will be conducted from the perspective of the NHS and personal social services. In Australia, the analysis will take the Australian health system and the patient's perspective. For the plan that follows, appropriate Australian measures will be included in the analyses of the Australian patient cohort.

Healthcare resource utilisation will be collected for each patient alongside the trial through a patient diary included as part of the CRF. Patients with an ongoing SSI at time of discharge will continue to complete the patient diary until healing of the SSI. Items of resource use will be costed using national sources and tariffs such as the Personal Social Services Research Unit ${ }^{19}$ and NHS reference cost databases ${ }^{20}$.

Generic health-related quality of life data will be collected using the EQ-5D-5L instrument at baseline and each follow-up assessment. Base-case analyses will be conducted using the crosswalk value sets for the EQ-5D-5 $\mathrm{L}^{21}$ with sensitivity analyses conducted using the EQ-5D-5L value set for England ${ }^{22}$. Quality adjusted life years (QALYs) will be calculated using the area under the curve approach, with regressionbased adjustment for baseline EQ-5D-5L score and minimisation variables.

A trial-based economic evaluation will take the form of a cost-utility analysis with results presented as incremental cost-utility ratios (ICURs). Data will be analysed on an intention to treat basis. Sensitivity analysis will consider the impact of missing data using appropriate techniques including multiple imputation. Deterministic and probabilistic sensitivity analysis will be undertaken to explore the robustness of the results to plausible variations in key assumptions and variations in the analytical methods used. Cost-effectiveness acceptability curves (CEACs) will be plotted to show the probability of the intervention being cost-effective considering a range of willingness to pay thresholds per additional QALY gained.

Full details will be included in the Health Economics Analysis Plan (HEAP).

\section{Adverse Events and Serious Adverse Events}

The safety profile of the SUNPDs used in this trial is well documented and patients undergoing emergency laparotomies are a highly morbid group that unfortunately inevitably incur a high number of adverse events. SUNRRISE will therefore only collect adverse events related to the SUNPD dressings such as a skin reaction to the applied dressing and pain/discomfort at the dressing site. These will be collected within the routine CRFs along with SSIs and other wound complications. The relatedness of the adverse event to the SUNPD dressing will be judged by the local principal investigator at each site.

Patients undergoing emergency laparotomies will often incur morbidity. Serious Adverse Events (SAEs) that do not require expedited reporting because they are expected and unrelated, and collected on the routine CRFs, have been identified. These are prolonged hospital stay or re-admission as a result of wound complications, anastomotic leak, intra-peritoneal collections (with or without intervention), thromboembolic events, infections not related to the wound (e.g. pneumonia, urinary tract 
infections), cardiac or central nervous system complications and paralytic ileus. Any other events that fulfil the usual definition of an SAE will be reported using a Serious Adverse Event form. In addition, specific complications that will always be reported on a Serious Adverse Event form are an enterocutaneous fistula, fascial dehiscence and death. The SAEs that are protocol excluded from reporting are any pre-planned hospitalisation, SAEs that are related to a pre-existing condition and SAEs that are related to symptoms or progression of the participant's disease.

Adverse events will be documented by sites and where applicable reported until 30 days post-surgery.

\section{Trial management}

The trial in the UK will be managed by the Birmingham Clinical Trials Unit (BCTU), with Australian sites managed by The University of Newcastle, Australia. Sites will be monitored centrally based on an agreed trial monitoring plan and on-site monitoring will take place if triggered. Any protocol amendments will be disseminated to local Principal Investigators and R\&D departments at participating sites. All trial data will be held securely at the University of Birmingham.

\section{Data management}

Data is collected using paper CRF and participant completed questionnaires/diaries. These are sent to BCTU and The University of Newcastle, Australia for transcription onto the SUNRRISE REDCap database. All missing and ambiguous data will be queried. Data Clarification Forms (DCF) will be sent to participating sites requesting missing data or clarification of inconsistencies or discrepancies. In all cases, it remains the responsibility of the Principal Investigator to ensure that the CRF has been completed correctly and that the data are accurate.

\section{Delivery network}

SUNRRISE was jointly designed by the North West Research Collaborative (NWRC) and the West Midlands Research Collaborative (WMRC), two trainee-led research groups. These are supported by the University of Birmingham Clinical Trials Unit (BCTU), the Birmingham Surgical Trials Consortium (BiSTC), the North West Surgical Trials Centre (NWSTC) and the SUNRRISE Trial Office at the University of Newcastle, Australia. Trainees designed and wrote the protocol and two trainees are co-chief investigators for the trial supported by senior surgeons and clinical trial methodologists from BCTU. The protocol was disseminated to potential participating centres in the UK and Australia through national research networks.

Trial delivery will be heavily supported by involvement of junior doctors at each site. Recruiting patients having an emergency laparotomy is a challenge and will require involvement of a large research team at each site with the aim of being able to recruit patients having surgery outside of normal working hours as effectively as those during the working day. Sites will be encouraged to develop communication channels within the teams to ensure that eligible patients are approached, enrolled and followed up. 


\section{Trial oversight}

Trial oversight will be provided by the trial management group, an independent DMC and independent TSC. Interim analyses of major outcome measures and safety data will be conducted and provided in strict confidence to the DMC.

\section{Results dissemination and authorship policy}

Results of this trial will be submitted for publication in a peer reviewed journal and presented at international conferences. The manuscript and presentations will be prepared by the TMGs.

The main results manuscript of the trial and any subsequent secondary analysis manuscripts using the data collected in the trial will be published under a corporate authorship policy. The authorship policy will closely mirror the suggestion published by the National Research Collaborative ${ }^{23}$. There will be no named authors in the main authorship line but individuals will be named within the paper and roles will be defined. All collaborators will be named and will be PubMed citable.

The datasets used and analysed during this study are available from the corresponding author on reasonable request once the main trial results have been published.

\section{Potential impact}

SUNRRISE is a large, pragmatic, international, multicentre, RCT which aims to provide robust evidence around the clinical and cost effectiveness of SUNPD at reducing SSI in patients undergoing an emergency laparotomy. As of July 2020, SUNRRISE is over halfway through recruitment. It is hoped the outcome of this trial could be used to provide robust evidence to direct dressing choice after emergency laparotomy in the future.

\section{References}

1 Pinkney TD, Calvert M, Bartlett DC, Gheorghe A, Redman V, Dowswell G, et al. Impact of wound edge protection devices on surgical site infection after laparotomy: multicentre randomised controlled trial (ROSSINI Trial). BMJ [Internet]. BMJ Publishing Group Ltd; 2013; 347. Available from: https://www.bmj.com/content/347/bmj.f4305

2 Uchino M, Ikeuchi H, Matsuoka H, Takahashi Y, Tomita N, Takesue Y. Surgical site infection and validity of staged surgical procedure in emergent/urgent surgery for ulcerative colitis. Int Surg [Internet]. International College of Surgeons; 2013; 98: 24-32. Available from: https://pubmed.ncbi.nlm.nih.gov/23438273

3 NELA Project Team. Third Patient Report of the National Emergency Laparotomy Audit. RCoA London, 2017

4 Symons NRA, Moorthy K, Almoudaris AM, Bottle A, Aylin P, Vincent CA, et al. Mortality in high-risk emergency general surgical admissions. BJS (British $J$ Surgery) [Internet]. 2013; 100: 1318-1325. Available from: 
https://bjssjournals.onlinelibrary.wiley.com/doi/abs/10.1002/bjs.9208

The National Emergency Laparoromy Audit. The second patient report of the National Emergency Laparotomy Audit. 2016.

Leaper DJ, Van Goor H, Reilly J, Petrosillo N, Geiss HK, Torres AJ, et al. Surgical site infection - a European perspective of incidence and economic burden. Int Wound J [Internet]. John Wiley \& Sons, Ltd; 2004 Dec 1; 1: 247273. Available from: https://doi.org/10.1111/j.1742-4801.2004.00067.x

Reddix RN, Leng XI, Woodall J, Jackson B, Dedmond B, Webb LX. The effect of incisional negative pressure therapy on wound complications after acetabular fracture surgery. J Surg Orthop Adv [Internet]. 2010; 19: 91-97. Available from: http://europepmc.org/abstract/MED/20727304

8 Pachowsky M, Gusinde J, Klein A, Lehrl S, Schulz-Drost S, Schlechtweg P, et al. Negative pressure wound therapy to prevent seromas and treat surgical incisions after total hip arthroplasty. Int Orthop [Internet]. 2012; 36: 719-722. Available from: https://doi.org/10.1007/s00264-011-1321-8

9 Costa ML, Achten J, Knight R, Bruce J, Dutton SJ, Madan J, et al. Effect of Incisional Negative Pressure Wound Therapy vs Standard Wound Dressing on Deep Surgical Site Infection After Surgery for Lower Limb Fractures

Associated With Major Trauma: The WHIST Randomized Clinical Trial. JAMA [Internet]. 2020 Feb 11; 323: 519-526. Available from:

https://doi.org/10.1001/jama.2020.0059

10 Allegranzi B, Zayed B, Bischoff P, Kubilay NZ, de Jonge S, de Vries F, et al. New WHO recommendations on intraoperative and postoperative measures for surgical site infection prevention: an evidence-based global perspective. Lancet [Internet] 2016 Dec; 16: 12: 288-303. Available from https://doi.org/10.1016/S1473-3099(16)30402-9

11 Sahebally SM, McKevitt K, Stephens I, Fitzpatrick F, Deasy J, Burke JP, et al. Negative Pressure Wound Therapy for Closed Laparotomy Incisions in General and Colorectal Surgery: A Systematic Review and Meta-analysis. JAMA Surg [Internet]. 2018 Nov 21; 153: e183467-e183467. Available from: https://doi.org/10.1001/jamasurg.2018.3467

12 Shiroky J, Lillie E, Muaddi H, Sevigny M, Choi WJ, Karanicolas PJ. The impact of negative pressure wound therapy for closed surgical incisions on surgical site infection: A systematic review and meta-analysis. Surgery [Internet]. 2020; 167: 1001-1009. Available from:

http://www.sciencedirect.com/science/article/pii/S0039606020300593

13 Allegranzi B, Zayed B, Bischoff P, Kubilay NZ, de Jonge S, de Vries F, et al. New WHO recommendations on intraoperative and postoperative measures for surgical site infection prevention: an evidence-based global perspective. Lancet Infect Dis [Internet]. 2016; 16: e288-e303. Available from: http://www.sciencedirect.com/science/article/pii/S1473309916304029

14 Norman G, Goh EL, Dumville JC, Shi C, Liu Z, Chiverton L, Stankiewicz M, Reid A. Negative pressure wound therapy for surgical wounds healing by primary closure. Cochrane Database of Systematic Reviews [Internet] 2020, Issue 6. Art. No.: CD009261. DOI: 10.1002/14651858.CD009261.pub6. Available from: https://www.ncbi.nlm.nih.gov/pmc/articles/PMC7389520/pdf/CD009261.pdf Kuper TM, Murphy PB, Kaur B, Ott MC. Prophylactic Negative Pressure Wound Therapy for Closed Laparotomy Incisions. A Meta-analysis of Randomized Controlled Trials. Ann Surg [Internet]. 2020; 271. 1. 67-74. Available from: 
https://journals.Iww.com/annalsofsurgery/Abstract/2020/01000/Prophylactic_N egative_Pressure_Wound_Therapy_for.12.aspx

16 Chan A-W, Tetzlaff JM, Gø̄tzsche PC, Altman DG, Mann H, Berlin JA, et al. SPIRIT 2013 explanation and elaboration: guidance for protocols of clinical trials. BMJ [Internet]. BMJ Publishing Group Ltd; 2013; 346. Available from: https://www.bmj.com/content/346/bmj.e7586

17 Daniel Dindo, MD, Nicolas Demartines, MD, and Pierre-Alain Clavien, Classification of Surgical Complications A New Proposal With Evaluation in a Cohort of 6336 Patients and Results of a Survey. Ann Surg. 2004 Aug; 240(2): 205-213. Available from:

https://www.ncbi.nlm.nih.gov/pmc/articles/PMC1360123/

18 Smith+Nephew. PICO 7 | Smith+Nephew Corporate [Internet]. [cited 2020 Jun 4]. Available from: https://www.smith-nephew.com/key-products/advancedwound-management/pico/pico-7/

19 Curtis L, Burns A. Unit Costs of Health and Social Care 2018 [Internet]. Canterbury; 2018. Available from: https://www.pssru.ac.uk/project-pages/unitcosts/unit-costs-2018/

20 Department of Health. National Schedule of Reference Costs 2017/2018 [Internet]. 2018. Available from: https://improvement.nhs.uk/resources/reference-costs/

21 National Institue for Health and Care Excellence. Position statement on use of the EQ-5D-5L value set for England (updated October 2019) [Internet]. Available from: https://www.nice.org.uk/about/what-we-do/ourprogrammes/nice-guidance/technology-appraisal-guidance/eq-5d-5I

22 Devlin NJ, Shah KK, Feng Y, Mulhern B, van Hout B. Valuing health-related quality of life: An EQ-5D-5L value set for England. Health Econ [Internet]. John Wiley \& Sons, Ltd; 2018 Jan 1; 27: 7-22. Available from: https://doi.org/10.1002/hec.3564

23 Blencowe N, Glasbey J, Heywood N, Kasivisvanathan V, Lee M, Nepogodiev $\mathrm{D}$, et al. Recognising contributions to work in research collaboratives: Guidelines for standardising reporting of authorship in collaborative research. Int J Surg [Internet]. 2018; 52: 355-360. Available from: http://www.sciencedirect.com/science/article/pii/S174391911731498X 
Table 1: Stop/Go criteria

\begin{tabular}{|c|c|c|c|c|}
\hline & Recruitment & Adherence & Drop-out \\
\hline \multicolumn{2}{|c|}{ Definition } & $\begin{array}{l}\text { Proportion of } \\
\text { recruitment target } \\
\text { achieved (aim is to } \\
\text { recruit } 70 \text { patients) }\end{array}$ & $\begin{array}{l}\text { Successful application } \\
\text { of the appropriate } \\
\text { dressing for at least } 24 \\
\text { hours }\end{array}$ & $\begin{array}{l}\text { Complete withdrawal } \\
\text { from the study, with no } \\
\text { further data collection } \\
\text { for the participant }\end{array}$ \\
\hline \multirow{2}{*}{ 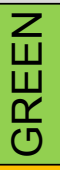 } & Criteria & $>70 \%$ & $>80 \%$ & $<20 \%$ \\
\hline & \multicolumn{4}{|c|}{ If all three criteria are met; continue the trial with protocol unchanged } \\
\hline \multirow{2}{*}{ 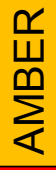 } & Criteria & $50-70 \%$ & $50-80 \%$ & $23-35 \%$ \\
\hline & \multicolumn{4}{|c|}{$\begin{array}{l}\text { If one or more of our amber criteria are met, then the study will need review to see what } \\
\text { changes (if any) could be made to improve whichever criteria are not at the "green" level }\end{array}$} \\
\hline \multirow{2}{*}{ 岂 } & Criteria & $<50 \%$ & $<50 \%$ & $>35 \%$ \\
\hline & \multicolumn{4}{|c|}{$\begin{array}{l}\text { If one or more of these criteria are met, we will discuss with the Trial Steering Committee } \\
\text { (TSC) and the funder regarding feasibility of the study continuing }\end{array}$} \\
\hline
\end{tabular}


Figure 1: Consent Process Flowchart

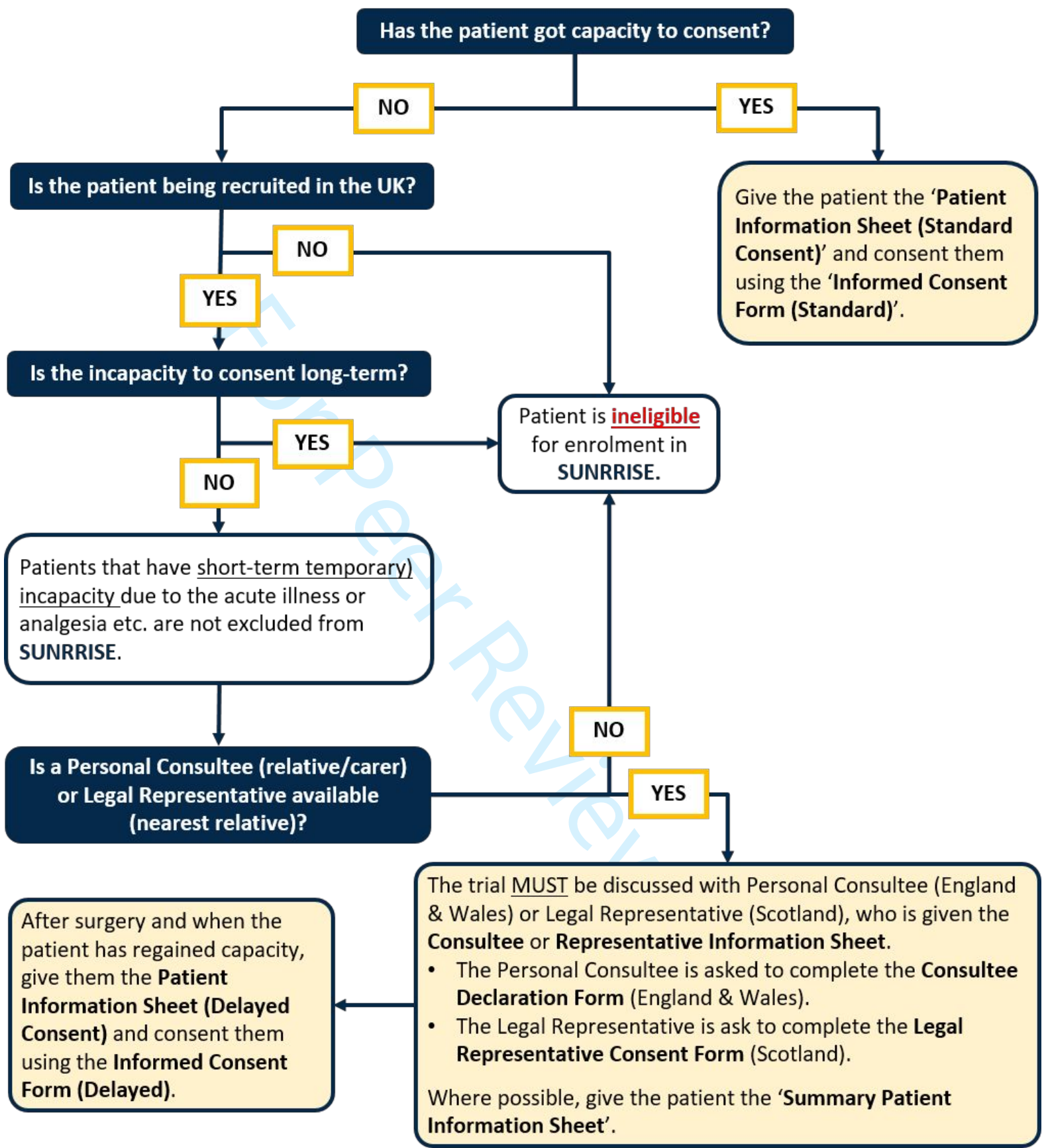




\section{Figure 2: Assessment schedule}

\begin{tabular}{|c|c|c|c|c|c|c|c|c|}
\hline \multirow{2}{*}{\multicolumn{2}{|c|}{$\begin{array}{l}\text { Activity/CRF } \\
\begin{array}{l}\text { Patient identification and } \\
\text { screening }\end{array}\end{array}$}} & Pre-theatre & In-theatre & $\begin{array}{l}\text { Day } 7 \text { post-op } \\
(-2 /+3 \text { days })\end{array}$ & $\begin{array}{l}\text { Day } 14 \text { post-op } \\
\text { ( } \pm 2 \text { days) }\end{array}$ & $\begin{array}{l}\text { Day } 21 \text { post-op } \\
\text { ( } \pm 2 \text { days) }\end{array}$ & $\begin{array}{l}\text { Day } 30 \text { post-op } \\
\text { (+ } 14 \text { days) }\end{array}$ & Day 30+ \\
\hline & & On-call surgical team & & & & & & \\
\hline \multirow{2}{*}{ 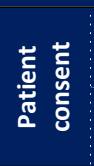 } & - Standard & $\begin{array}{l}\text { Patient \& Member of the } \\
\text { research team }\end{array}$ & & & & & & \\
\hline & $\begin{array}{l}\text { - Delayed } \\
\text { (UK only) }\end{array}$ & $\begin{array}{l}\text { Consultee/Representative } \& \\
\text { Member of the research team }\end{array}$ & \multicolumn{6}{|c|}{ Patient \& Member of the research team when capacity regained } \\
\hline \multicolumn{2}{|c|}{ Randomisation form } & $\begin{array}{l}\text { Started pre-theatre by the } \\
\text { research team }\end{array}$ & $\begin{array}{l}\text { Surgeon or member of } \\
\text { the research team }\end{array}$ & & & & & \\
\hline \multicolumn{2}{|c|}{ In-Theatre form } & & $\begin{array}{l}\text { Ideally an operating } \\
\text { surgeon, or member } \\
\text { of the research team }\end{array}$ & & & & & \\
\hline \multicolumn{2}{|c|}{$\begin{array}{l}\text { Wound Assessment Day } 7 \\
\text { or on Discharge (if } \\
\text { sooner) }{ }^{1,2}\end{array}$} & & & $\begin{array}{l}\text { Member of the } \\
\text { research team }\end{array}$ & & & & \\
\hline \multicolumn{2}{|c|}{ EQ-5D-5L } & Completed by the participant & & $\begin{array}{l}\text { Completed by } \\
\text { the participant }\end{array}$ & $\begin{array}{l}\text { Completed by the } \\
\text { participant }\end{array}$ & $\begin{array}{l}\text { Completed by the } \\
\text { participant }\end{array}$ & Completed by the participant & \\
\hline \multicolumn{2}{|l|}{ SF-12 } & Completed by the participant & & $\begin{array}{l}\text { Completed by } \\
\text { the participant }\end{array}$ & 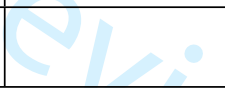 & & Completed by the participant & \\
\hline \multicolumn{2}{|c|}{ Patient diary } & & & \multicolumn{4}{|c|}{$\begin{array}{l}\text { Completed daily by the participant following discharge from hospital until they undergo the Day } \\
30 \text { wound review }\end{array}$} & $\begin{array}{l}\text { Patients to continue with a diary if } \\
\text { they have an ongoing SSI }\end{array}$ \\
\hline \multicolumn{2}{|c|}{$\begin{array}{l}\text { Bluebelle wound healing } \\
\text { questionnaire }\end{array}$} & & & & & & \begin{tabular}{|l|} 
Completed by the participant \\
independently, and then by the \\
participant with a blinded member of \\
the research team reviewing wound
\end{tabular} & \\
\hline \multicolumn{2}{|c|}{$\begin{array}{l}\text { Wound Assessment Day } \\
30^{1}\end{array}$} & & & & & & $\begin{array}{l}\text { Completed by a blinded member of the } \\
\text { research team as an in-person or } \\
\text { remote (video) review }\end{array}$ & \\
\hline \multicolumn{2}{|c|}{ SAE reporting } & & \multicolumn{5}{|c|}{$\begin{array}{l}\text { All serious adverse events by member of the research team using SAE form or wound assessment CRF if excluded from } \\
\text { expedited reporting }\end{array}$} & $\begin{array}{l}\text { Related serious adverse events } \\
\text { only }\end{array}$ \\
\hline \multicolumn{2}{|c|}{ Return to theatre form } & & \multicolumn{5}{|c|}{ Member of the research team for any return to theatre following patient returning to theatre } & \\
\hline \multicolumn{2}{|c|}{$\begin{array}{l}\text { PI Declaration form for } \\
\text { CFR data }\end{array}$} & & & & & & & $\begin{array}{l}\text { Completed by Pl at the end of each } \\
\text { participant's involvement }\end{array}$ \\
\hline
\end{tabular}

Assessment of pain undertaken by a member of the research team by asking the participant and recording the response on the CRF participant's involvemen

${ }^{2}$ Score of patient acceptability of dressing undertaken by a member of the research team by asking the participant and recording the response on the CRF 


\section{Appendix 1: Collaborating authors}

Writing group: Richard Wilkin (University of Birmingham), Hamish Clouston (The Christie NHS Foundation Trust), Peter Coe (Leeds Teaching Hospital NHS Trust), Rui Duarte (University of Liverpool), Martyn Stott (Manchester University NHS Foundation Trust), Peter Pockney (University of Newcastle, Australia), Natasha Egoroff (University of Newcastle), Samir Mehta (University of Birmingham Clinical Trials Unit), James Brown (University of Birmingham Clinical Trials Unit), Laura Magill (University of Birmingham Clinical Trials Unit), Natalie Ives (University of Birmingham Clinical Trials Unit), Rebecca Fish (Stockport NHS Foundation Trust), James Glasbey (University of Birmingham), Sarah Duff (Manchester University NHS Foundation Trust), Thomas Pinkney (University Hospitals Birmingham NHS Foundation Trust)

Patient Representative: Elisabeth Johnstone

Protocol development:

Australia- Hossein Afzali (Flinders University), Thomas Arthur (Austin Health), James Brown (University of Birmingham Clinical Trials Unit), Amanda Dawson (University of Newcastle), Nagendra N Dudi-Venkata (Royal Adelaide Hospital), Natasha Egoroff (University of Newcastle), Madelyn Gramlick (John Hunter Hospital), Yick Ho Lam (Flinders Medical Centre), Laura Magill (University of Birmingham Clinical Trials Unit), Vijayaragavan Muralidharan (University of Melbourne), Sean SW Park (Gosford Hospital), Peter Pockney (University of Newcastle), Toby Richards (University of Western Australia), Tarik Sammour (University of Adelaide), Bree Stephensen (Sunshine Coast University Hospital), Sean Stevens (Austin Health), Uyen Giao Vo (Fiona Stanley Hospital), David Watson (Flinders University), Richard Wilkin (University of Birmingham)

UK- James Brown (University of Birmingham Clinical Trials Unit), Peter Coe (Leeds Teaching Hospital NHS Trust), Hamish Clouston (The Christie NHS Foundation Trust), Tony Coffey (Liverpool and North West Surgical Trials Centre), Nicola Cook (Liverpool and North West Surgical Trials Centre), Rui Duarte (University of Liverpool), Sarah Duff (Manchester University NHS Foundation Trust), Rebecca Fish (Stockport NHS Foundation Trust), Paula Ghaneh (University of Liverpool), James Glasbey (University of Birmingham), Alan Haycox University of Liverpool), Natalie Ives (University of Birmingham Clinical Trials Unit), Manjinder Kaur (University of Birmingham Clinical Trials Unit), Laura Magill (University of Birmingham Clinical Trials Unit), Samir Mehta (University of Birmingham Clinical Trials Unit), Thomas Pinkney (University Hospitals Birmingham NHS Foundation Trust), Kellie Platt (Liverpool and North West Surgical Trials Centre), Hema Sehkar (Salford Royal NHS Foundation Trust), Yash Sinha, (University of Birmingham), Martyn Stott (Manchester University NHS Foundation Trust), Kathryn Waite (Worcestershire Acute Hospitals NHS Trust), Richard Wilkin (University of Birmingham) 


\section{Appendix 2: Site activity flowchart/schematic}

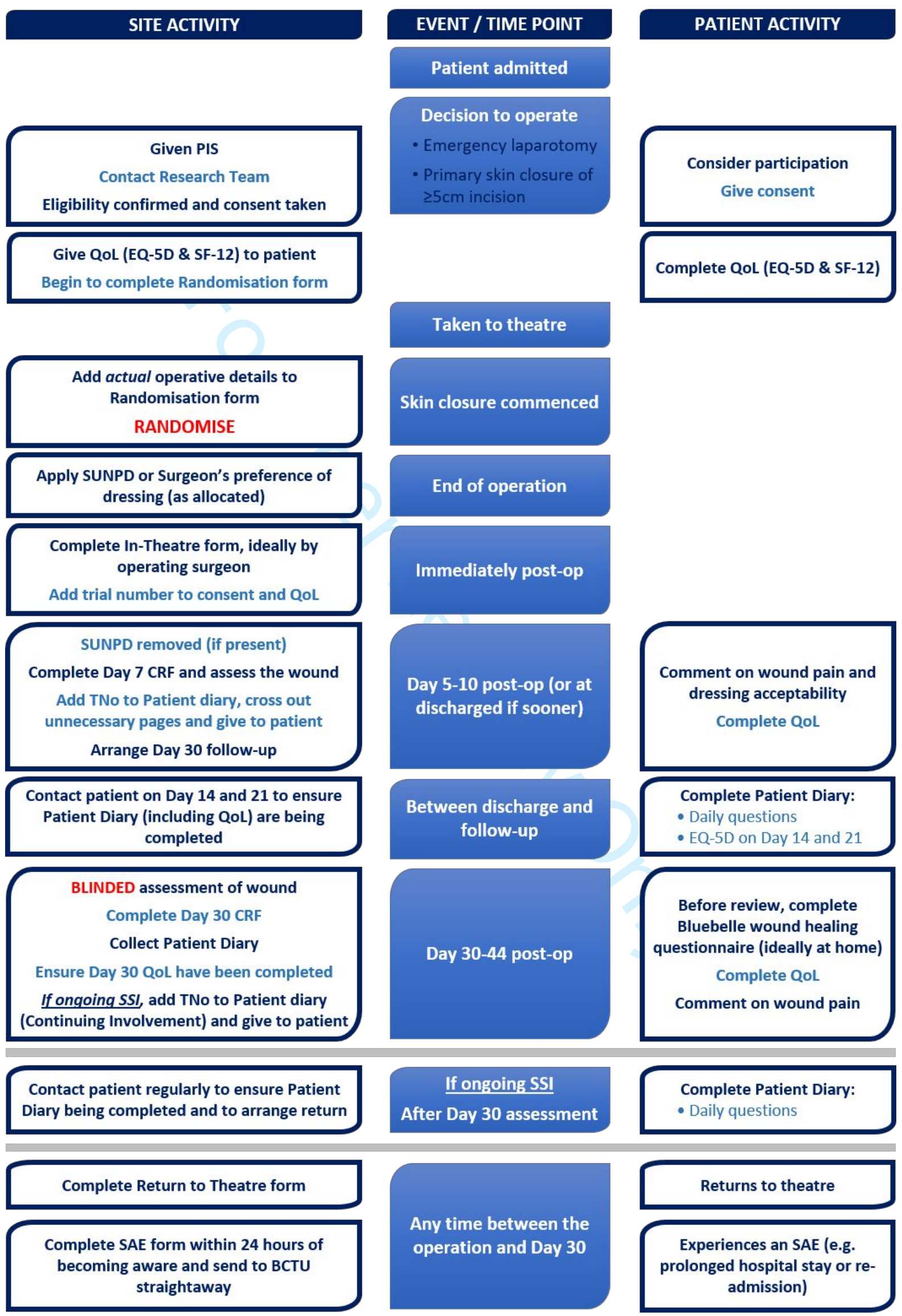


Appendix 3: World Health Organisation trial registration dataset table

\begin{tabular}{|c|c|}
\hline Data category & Information \\
\hline Primary registry and trial identifying number & ISRCTN number: 17599457 \\
\hline Date of registration in primary registry & 28/09/2018 \\
\hline Secondary identifying numbers & ACTRN12619000496112 \\
\hline Source(s) of monetary or material support & $\begin{array}{l}\text { RfPB program of NIHR, ICTC scheme of } \\
\text { MRFF }\end{array}$ \\
\hline Primary sponsor & University of Birmingham, UK \\
\hline Secondary sponsor(s) & University of Newcastle, Australia \\
\hline Contact for public queries & $\begin{array}{l}\text { James Brown, Trial Manager } \\
\text { sunrrise@trials.bham.ac.uk }\end{array}$ \\
\hline Contact for scientific queries & $\begin{array}{l}\text { Richard Wilkin, Academic Clinical Lecturer } \\
\text { r.wilkin@bham.ac.uk }\end{array}$ \\
\hline Public title & $\begin{array}{l}\text { Single Use Negative pRessure dressing for } \\
\text { Reduction In Surgical site infection following } \\
\text { Emergency laparotomy: The SUNRRISE } \\
\text { Trial }\end{array}$ \\
\hline Scientific title & $\begin{array}{l}\text { An international pragmatic randomised } \\
\text { controlled trial to compare a single use } \\
\text { negative pressure dressing versus a } \\
\text { surgeon's preference of dressing to reduce } \\
\text { the incidence of surgical site infection } \\
\text { following emergency laparotomy: the } \\
\text { SUNRRISE Trial }\end{array}$ \\
\hline Countries of recruitment & United Kingdom, Australia \\
\hline Health condition(s) or problem(s) studied & $\begin{array}{l}\text { Surgical site infection after emergency } \\
\text { laparotomy }\end{array}$ \\
\hline Intervention(s) & Single use negative pressure dressings \\
\hline Key inclusion and exclusion criteria & $\begin{array}{l}\text { Inclusion criteria } \\
\text { - Patients undergoing emergency } \\
\text { (non-elective) laparotomy } \\
\text { - Procedures with an incision of at } \\
\text { least } 5 \mathrm{~cm}\end{array}$ \\
\hline
\end{tabular}




\begin{tabular}{|c|c|}
\hline & $\begin{array}{l}\text { - Operations where the skin is closed } \\
\text { primarily } \\
\text { - Patients aged at least } 16 \text { years (18 } \\
\text { years in Australia) } \\
\text { - Patients able to provide informed } \\
\text { consent, or consultee/representative } \\
\text { provides assent/consent if a patient } \\
\text { temporarily lacks capacity } \\
\text { - Patients willing and able to attend } \\
\text { follow-up at } 30 \text { days post-op } \\
\text { Exclusion criteria } \\
\text { - Abdominal surgery within the } \\
\text { preceding three months of } \\
\text { randomisation } \\
\text { - Expected return to theatre for } \\
\text { reopening of the laparotomy wound } \\
\text { within } 30 \text { days }\end{array}$ \\
\hline Study type & $\begin{array}{l}\text { International, multicentre, assessor blinded, } \\
2 \text { arm, superiority randomised controlled } \\
\text { trial }\end{array}$ \\
\hline Date of first enrolment & $18 / 12 / 2018$ \\
\hline Target sample size & 840 \\
\hline Recruitment status & Ongoing \\
\hline Primary outcome(s) & $\begin{array}{l}\text { Surgical site infection at } 30 \text { days post- } \\
\text { procedure }\end{array}$ \\
\hline Key secondary outcomes & $\begin{array}{l}\text { Length of hospital stay, wound } \\
\text { complications, readmission rate, quality of } \\
\text { life, cost effectiveness, patient acceptability }\end{array}$ \\
\hline
\end{tabular}

